\title{
Alveolar macrophage immaturity in infants and young children
}

\author{
J. Grigg*, J. Riedler*, C.F. Robertson*, W. Boyle ${ }^{\#}$, S. Uren ${ }^{\#}$
}

\begin{abstract}
Alveolar macrophage immaturity in infants and young children. J. Grigg, J. Riedler, C.F. Robertson, W. Boyle, S. Uren. (C)ERS Journals Ltd 1999

ABSTRACT: Very little is known about alveolar macrophage (AM) immunological function in early childhood. Using nonbronchoscopic bronchoalveolar lavage (BAL), this study sought to compare the proportion, number, and function of AM between very young and older children.

BAL fluid (BALF) leukocyte parameters were determined in 63 children, and data divided into 3 age groups: group $1(<2$ yrs), group $2(\geq 2-\leq 5$ yrs) and group 3 ( $\geq 6$ $\leq 17$ years). In a further subgroup of children, AM function and immune receptor expression were assessed, and data categorized into two age groups: $<2$ yrs and $\geq 2$ yrs of age.

Compared to groups 2 and 3, the AM percentage in the BAL in group 1 was significantly increased (median: $98 \%$ versus $92 \%$ and $91 \%$ ), as was the albuminadjusted AM concentration. AM from children $<2$ yrs expressed less human leukocyte antigen (HLA)-DR (versus $\geq 2$ yrs of age), were less effective in reducing nitro blue tetrazolium, and released less interleukin (IL)-1 and tumour necrosis factor on lipopolysaccharide stimulation. There was no difference in release of IL-6, expression of intercellular adhesion molecule-1 (CD54), and AM stimulation of allogeneic T-cells, between children $<2$ yrs and $\geq 2$ yrs of age.

It was concluded that the capacity of alveolar macrophage to stimulate T-cells is not enhanced in early childhood, and that immaturity of alveolar macrophage function may contribute to an increased susceptibility to respiratory infections in this age group.

Eur Respir J 1999; 14: 1198-1205.
\end{abstract}

*Dept of Thoracic Medicine, Royal Children's Hospital Melbourne, Victoria, Australia. "University of Melbourne, Dept of Microbiology and Immunology Royal Parade, Parkville, Victoria, Australia.

Correspondence: J. Grigg, The Leicester Children's Asthma Centre, University of Leicester School of Medicine, Robert Kilpatrick Clinical Sciences Building, Leicester Royal Infirmary, PO Box 65, Leicester LE2 7LX, UK. Fax: 44116 2523282

Keywords: Alveolar macrophage bronchoalveolar lavage

children

immune receptor

T-cells

Received: December 291998

Accepted after revision July 151999

J. Grigg was supported by a ScaddingMorriston-Davies Fellowship (UK).
Young children are particularly susceptible to lower respiratory tract infections [1], and it has been speculated that they are at increased risk of developing T-cell sensitization to inhaled antigens [2]. The period necessary to achieve immune competence in the human lung may therefore be a time span of vulnerability for both pulmonary allergic sensitization and infection. Alveolar macrophages (AM) are the most numerous immune cells in the alveolar air-tissue interface, and are among the first immune cells to encounter material inhaled into the alveoli [3]. The antimicrobial functions of AM include phagocytosis, oxidant mediated microbial killing, and the recruitment of leukocytes by pro-inflammatory cytokines. Studies in the rhesus monkey have suggested that AM in the neonatal lung are deficient in a range of antimicrobial activities [4], although no parallel studies have been performed in healthy young children. AM are also important in controlling responses to inhaled antigens. AM express human leukocyte antigen (HLA)-DR and have the capacity to act as antigen presenting cells to pulmonary T-lymphocytes $[5$, 6]. However, compared to monocytes, AM from adult subjects are poor stimulators of antigen-specific responses in vitro, and indeed suppress these activities [6]. There is no data on whether the ability of AM to stimulate Tcells is different in early childhood. However, the increased susceptibility in young children to respiratory infections, and an increased vulnerability to pulmonary antigen sensitization could be associated with a reduced ability of AM to remove and kill microbes, and an increased capacity to stimulate pulmonary T-cells.

To identify how development influences AM antimicrobial and immune function in children, AM have been studied from healthy noninfected children aged between 7 days and 17 yrs who were intubated for elective surgery. $\mathrm{AM}$ and other alveolar leukocytes were collected prior to surgery by a nonbronchoscopic bronchoalveolar lavage (BAL) technique as previously described [7].

\section{Subjects and methods}

\section{Subjects}

Children between 7 days and 17 yrs of age intubated for elective surgery were eligible for BAL. The exclusion criteria were: premature birth, chronic respiratory pathology, chronic systemic disease, atopic symptoms, acute respiratory symptoms in the preceding 8 weeks, and the isolation of bacterial or viral pathogens from the BAL fluid (BALF). The study was approved by the Ethics in Human Research Committee of the Royal Children's Hospital and written informed parental consent was obtained. 


\section{Bronchoalveolar lavage}

BAL was performed before surgery by a single operator using a nonbronchoscopic BAL technique [7]. Immediately after intubation, and with the child's head turned to the left, a straight $60 \mathrm{~cm}$ end hole suction catheter (Vygon S.A., Ecouen, France) was inserted into the endotracheal tube (ET) through a right-angle swivel bronchoscope connector (Mallinckrodt Medical Pty. Ltd., Mount Waverly, Australia). Catheter size was adjusted to the ET diameter (size 6 French catheter for size $\leq 3.0$ ET, 7 French catheter for size 3.5 ET, 8 French catheter for size 3.5-5.0 ET, 10 French catheter for size $\geq 5.5$ ET). The catheter was advanced until wedged, $1 \mathrm{~mL} \cdot \mathrm{kg}$ body weight ${ }^{-1}$ of saline at room temperature was instilled, and BALF immediately aspirated into a suction trap using $20 \mathrm{kPa}(150$ $\mathrm{mmHg}$ ) negative pressure and a three-way tap. With the catheter remaining wedged in situ, two further aliquots of $1 \mathrm{~mL} \cdot \mathrm{kg}^{-1}$ saline were instilled and aspirated. Pooled BALF was placed on ice and $0.5 \mathrm{~mL}$ aliquots cultured for respiratory viruses, aerobic and anaerobic bacteria. All potential bacterial pathogens and commensuals of the upper respiratory tract were identified and reported. Viral immunofluorescence was performed on cells concentrated by centrifugation. Immediately after collection, the concentration of leukocytes was determined by haemocytometer and viability determined by exclusion of a $0.04 \%$ solution of trypan blue. Ciliated epithelial cells in the haemocytometer chamber were not counted. Unfiltered BALF was cytocentrifuged at $91 \times g$ for 5 min (Cytospin 2, Shandon Products Ltd. Runcorn, UK), stained with Wilson's reagents and examined under oil microscopy ( $\times 1000$ magnification). BALF leukocyte differentials were determined by counting $\geq 300$ leukocytes. The proportion of ciliated epithelial cells (as a percentage of all nucleated cells) was determined on a separate count of $\geq 100$ stained nucleated cells. Unfixed cytocentrifuge cell preparations were air dried for $2 \mathrm{~h}$ and stored with desiccant at $-20^{\circ} \mathrm{C}$ for immunocytochemistry. BALF supernatant was obtained by centrifugation of BALF at 1000 $\times g$ for $10 \mathrm{~min}\left(4^{\circ} \mathrm{C}\right)$ and $8000 \times g$ for a further $3 \mathrm{~min}$ $\left(4^{\circ} \mathrm{C}\right)$. Aliquots of particulate free BALF were stored at $-70^{\circ} \mathrm{C}$. Using this protocol it had previously been determined that the total number of leukocytes recovered by BAL range from $1 \times 10^{5}$ in the youngest infant to $2 \times 10^{6}$ in adolescents.

\section{Bronchoalveolar lavage fluid albumin and urea}

Albumin and urea assays on BALF were performed in duplicate using a Roche Cobas centrifugal analyser (Roche diagnostics, Basel, Switzerland). Albumin was determined by immunoturbidometry with an assay linear dynamic range $0.005-0.8 \mathrm{mg} \cdot \mathrm{mL}^{-1}$. BALF urea was measured using a commercially available kit (Sigma UV-66; Sigma Chemical Co., St. Louis, MO, USA.). The kit was modified by increasing the sample volume to $50 \mu \mathrm{L}$, decreasing the reagent volume to $250 \mu \mathrm{L}$, and increasing the assay duration to $6 \mathrm{~min}$. The linear dynamic range for urea was 18 $500 \mu \mathrm{mol} \cdot \mathrm{L}^{-1}$.

The inter-assay coefficient of variation for both assays was $<9 \%$. Blood samples were not routinely obtained for ethical reasons. If blood could be sampled through the int- ravenous access used for induction of anaesthesia, plasma urea was determined using routine hospital biochemical techniques, and the volume of alveolar epithelial lining fluid (ELF) was estimated using the urea dilution technique [8].

\section{Cell size analysis}

Relative cell size (area) was determined by image analysis of cytospun BALF, previously stained with Wilson's reagents, using the Video Pro 32 Image Analysis System (Leading Edge Technology, Bedford Park, South Australia). Data are presented as the mean area of 200 cells measured for each child.

\section{Preservation of alveolar macrophages}

The sporadic availability of subjects, and the small numbers of cells per sample, made it an essential feature of this study that cell samples for functional analysis (cytokine release and T-cell stimulation) were processed within $80 \mathrm{~min}$ of collection and frozen under a well established protocol. Briefly, AM were pelleted and resuspended in Roswell Park Memorial Institute (RPMI) 1640 medium supplemented with $25 \%$ fetal calf serum (FCS) and $10 \%$ dimethyl sulphoxide and frozen at $2 \times 10^{5}-1 \times 10^{6}$ cells $\cdot \mathrm{mL}^{-1}$ in liquid nitrogen. In order to avoid possible inter-assay variations which might have occurred if sporadic samples were tested singly at the time of collection, it was decided to store samples using standard cryopreservation methods. Additionally, in neonates the number of cells were so low that only one vial of cells was stored. Since a number of assays had to be performed of AM phenotype and function, they had to be undertaken simultaneously following thawing to prevent complications induced by refreezing. To further minimize interassay variations, samples were thawed in batches for testing such that each batch had a matched age group distribution. Every assay included internal controls comprising aliquots of adult monocytes from a single donor stored in liquid nitrogen in multiple vials. It had previously been established that these storage conditions failed to induce any significant change in the results of similar function assays performed on peripheral blood monocytes and lymphocytes. Upon thawing, AM were washed three times, and suspended in RF10 ( RPMI with penicillin, streptomycin, glutamine, and $10 \%$ FCS). As an internal control all phenotypic and functional studies included a sample of adult monocytes prepared by counter-current elutriation as previously described [9], and stored in aliquots in liquid nitrogen. AM were collected for functional studies only if the timing of BAL was optimal for processing, and it was envisaged that AM from only a random subgroup of children undergoing BAL would be studied.

\section{Image analysis}

Results of immunocytochemistry and nitro blue tetrazolium (NBT) reduction were analysed by image analysis using the Video-Pro 32 Image Analysis System (Leading Edge Technology). Two parameters were assayed, the 
"integrated optical density" (IOD) in which the total colour development over each individual AM is assessed (a measurement analogous to that of fluorescence intensity using flow cytometry), and "density", the density per unit area (a parameter independent of cell area). These two methods in combination determine whether an increase in IOD is due to increased cell size. Measurements of IOD and density were performed on at least 200 cells for each individual sample.

\section{Immunocytochemical staining}

Stored cytocentrifuged cell preparations were warmed to room temperature, and fixed in ice-cold paraformaldehyde/ lysine/periodate solution. AM were stained using the four layer immunoperoxidase technique previously described by HANCOCK et al. [10]. Cytospun cells were reacted with mouse monoclonal antibodies to human leukocyte antigen (HLA)-DR, (Becton Dickinson, San Jose, CA, USA), CD54 (IH4; a kind gift from A. Boyd, Walter and Elisa Hall Institute of Medical Research, Melbourne, Australia), CD14 (3C10; American Type Culture Collection, Rockville, MD, USA) and CD68 (Dako (Australia) Pty, Botany, Australia). Control isotype antibodies were also employed. Second stage antibodies were as follows; goat antimouse immunoglobulin (Ig) (Sigma, St. Louis, MO, USA), rabbit antigoat Ig (Dako), goat peroxidase antiperoxidase (Dako). Density was measured using the Video Pro 32 Image Analysis System and data were presented as the total density over the whole cell (IOD) in arbitrary units of oxidized diaminobenzidine measured in 200 cells for each child.

\section{Control of endotoxin}

Reagents and media were selected so that, when employed their endotoxin levels were $<10 \mathrm{pg} \cdot \mathrm{mL}^{-1}$ when tested by the Limulus Amoebocyte Assay (Associates of Cape Cod, Falmouth, MA, USA). Plastics were supplied pyrogen-free and glassware was heated to $180^{\circ} \mathrm{C}$ for $5 \mathrm{~h}$ before use.

\section{Reduction of nitro blue tetrazolium salts}

This was performed by the method of Rook et al. [11]. AM were suspended to $1 \times 10^{5}$ cells $\cdot \mathrm{mL}^{-1}$ in RPMI. An equal volume of NBT (2.5 $\mathrm{mg} \cdot \mathrm{mL}^{-1}$, Sigma) together with phorbol myristic acetate $\left(200 \mathrm{ng} \cdot \mathrm{mL}^{-1}\right.$, Sigma) in phosphate buffered saline (PBS) was added and incubated for $30 \mathrm{~min}$ at $37^{\circ} \mathrm{C}$. Suspensions were cytocentrifuged and relative optical density of AM accumulating the purple formazan end product was measured by image analysis.

Interleukin-1, interleukin-6 and tumour necrosis factor release

AM were cultured at $10^{5} \mathrm{~mL}^{-1}$ in RF10 with or without the addition of $1 \mu \mathrm{g} \cdot \mathrm{mL}^{-1}$ lipopolysaccharide (LPS) ( $\mathrm{Sal}$ monella minnesota, Sigma) for $24 \mathrm{~h}$. Supernatant fluids were harvested, and stored at $-20^{\circ} \mathrm{C}$ for assay of induced cytokines. Assays for interleukin (IL)-1, (IL)-6 and tumour necrosis factor (TNF) were performed as previously described [12-14], but miniaturized by the use of Terasaki trays, to which $10 \mu \mathrm{L}$ of test sample together with $10 \mu \mathrm{L}$ of indicator cells was added. IL-1 activity in cell supernatants was measured by the method of GEARING et al. [12] which utilized the release of IL-2 from stimulated EL4.NOB1 cells; IL-2 (stimulated by IL-1) was assayed by proliferation of a cytotoxic T-lymphocyte cell line (CTLL); IL-6 was assayed by proliferation of murine Bcell hybridoma (7TD1) cells [13] and TNF was assayed by cytotoxicity for actinomycin D treated WEHI 164 cells [14]. At the end of the relevant incubation periods, 2 $\mu \mathrm{L}$ of 3-(4,5-dimethylthiazole-2-yl)-2,5-diphenyltetrazolium bromide (MTT, $10 \mathrm{mg} \cdot \mathrm{mL}^{-1}$; Sigma) was added to each well and trays incubated a further $3 \mathrm{~h}$, whereupon 15 $\mu \mathrm{L}$ supernatant fluid was removed and replaced with 10 $\mu \mathrm{L}$ lysing buffer [15]. Trays were left at room temperature overnight for complete solubilization of reduced formazan crystals. Colour development was measured as the percentage of light transmitted using the Leitz MPV Photometer and microscope system (Leica Instruments Pty, North Ride, Australia) and a $545 \mathrm{~nm}$ filter, with the medium control set at $100 \%$ light transmission. Recombinant (rh) cytokines (rhIL-1, $1 \times 10^{8} \mathrm{U} \cdot \mathrm{mg}^{-1}$; rhIL-2, $2 \times$ $10^{6} \mathrm{U} \cdot \mathrm{mg}^{-1}$ (Boehringer Mannheim, Australia Pty, Castle Hill, Australia); rhIL-6, $2 \times 10^{7} \mathrm{U} \cdot \mathrm{mg}^{-1}$ (Amersham, Amersham, UK); rhTNF- $\alpha$, Boehringer Mannheim, $1 \times 10^{8}$ $\mathrm{U} \cdot \mathrm{mg}^{-1}$ ) were included in the assays.

\section{Preparation of alveolar macrophages and responder $T$ - cells}

Responder T-cells were prepared as previously described from normal adult donors [9]. Briefly, peripheral blood mononuclear cells (PBMC) were separated from buffy coats (Red Cross Blood Bank, Victoria, Australia) by centrifugation over a Ficoll / Hypaque density barrier. Tcells were isolated from monocyte depleted fractions of PBMC prepared by counter current elutriation, and further purified by treatment with antibodies to HLA-DR (FMC4, a generous gift from H. Zola, FMC, South Australia), CD14 (3C10), antihuman IgG (Dako) and complement (prepared from a young rabbit in this laboratory). Prior to their addition to T-cell stimulation assays, AM were treated with $50 \mu \mathrm{g} \cdot \mathrm{mL}^{-1}$ mitomycin C (Sigma) for $30 \mathrm{~min}$ at $37^{\circ} \mathrm{C}$, washed three times in RF10 and resuspended to $1 \times 10^{6} \mathrm{~mL}^{-1}$. They were serially diluted and added to Terasaki trays (Nunc, Kamstrup, Roskilde) in triplicate in $10 \mu \mathrm{L}$ volumes.

\section{Alveolar macrophage stimulation of T-cell proliferation}

T-cells $\left(2 \times 10^{4}\right.$ cells $\left.\cdot 10 \mu \mathrm{L}\right)$ were added to serial dilutions of AM in inverted humidified Terasaki trays, so that the T-cell:AM ratio was 1:0.5, 1:0.25, 1:0.13, 1:0.06, 1:0.03 and 1:0.015. Trays were incubated for 6 days at $37^{\circ} \mathrm{C}$ in $\mathrm{CO}_{2}$. Eighteen hours before termination of cultures, $\left.1.85 \times 10^{7} \mathrm{~Bq} 0.5 \mu \mathrm{Ci}\right)$ tritiated thymidine $\left(\left[{ }^{3} \mathrm{H}\right] \mathrm{TdR}\right)$ (Amersham) was added. Conditions of culture and harvest were as described by O'BRIEN et al. [16]. Data were expressed as net counts per minute (CPM); mean and standard deviation of triplicate cultures, and responses were expressed for simplicity as the maximal proliferation achieved at any of the T-cell:AM ratios assayed. 
Alveolar macrophage stimulation of CD3-activated Tcell proliferation

Terasaki trays were coated with $8 \mathrm{ng} \cdot$ well protein A purified OKT3 (American Type Culture Collection) monoclonal antibody $(\mathrm{mAb})$ overnight at $4{ }^{\circ} \mathrm{C}$, washed three times in PBS, and blocked with RF10. AM were treated as described above, and graded doses added to the trays together with $10^{3}$ T-cells (total volume $20 \mu \mathrm{L}$ ), so that the ratio of T-cells:AM was 1:10, 1:5, 1:2,5, 1:1.25, 1:0.6 and $1: 0.3$. Cultures were incubated in the upright position for $80 \mathrm{~h}$. After the addition of $\left[{ }^{3} \mathrm{H}\right] \mathrm{TdR}$ for the final $18 \mathrm{~h}$ of culture, $10 \mu \mathrm{L}$ supernatant fluid was removed from each well and replaced with $10 \mu \mathrm{L}$ Sarkosyl buffer $(20 \%$ Sarkosyl in $30 \mathrm{mM}$ Tris and $100 \mathrm{mM}$ ethylenediamine tetraacetate, $\mathrm{pH} \mathrm{8)}$ and the trays harvested. Data were expressed as described above.

\section{Statistical analysis}

Variation between multiple groups was tested by Kruskal-Wallis analysis of variance. Differences between individual groups were tested by Mann-Whitney U-test. Stepwise multivariate linear regression analysis was performed after normalization of residuals by $\log _{10}$ transformation of the dependent and independent continuous variables. A value of $p<0.05$ was considered to be statistically significant throughout. Analysis was performed using a statistical package for microcomputers (SPSS Inc., Chicago, IL, USA).

\section{Results}

Children were categorized into three age groups for analysis of BALF leukocyte parameters: infants and youngest children $\leq 2$ yrs (group 1, $n=30$ ), preschool children $\geq 2-\leq 5$ yrs (group $2, n=17$ ), and school age children $\geq 6$ $\leq 17$ yrs (group 3, $n=16$ ) (fig. 1). Children were recategorized into $<2$ and $\geq 2$ yrs of age for functional and immunocytochemical studies, where subject numbers were limited to $11-18$.

There were no significant differences between groups 1 , 2 and 3 for: the percentage of instilled volume recovered; the percentage of BALF ciliated epithelial cells; and the BALF albumin concentration. There was no age-associated difference in the percentage of ELF recovered, and ELF albumin concentration.

\section{Bronchoalveolar lavage fluid leukocytes}

BALF leukocyte viability was $>90 \%$. There was no difference between groups 2 and 3 for any BALF leukocyte parameter. The AM\% in the differential cell count was significantly increased in group $1(\mathrm{p}<0.0001$ versus group 2 , or group 3; fig. 2a). The albumin-adjusted AM was also higher in group $1(\mathrm{p}<0.05$ versus group $2, \mathrm{p}<0.01$ versus group 3; fig. 2b). Multivariate analysis confirmed an independent negative correlation of age with the AM percentage, and the albumin-adjusted AM concentration, after adjusting for sex, previous wheeze, parental atopy, and percentage of BALF returned.

The BALF lymphocyte percentage was significantly decreased in group 1 ( $\mathrm{p}<0.0001$ versus group 2 or group 3$)$ (fig. $2 \mathrm{c}$ ), as was the albumin-adjusted lymphocyte con-
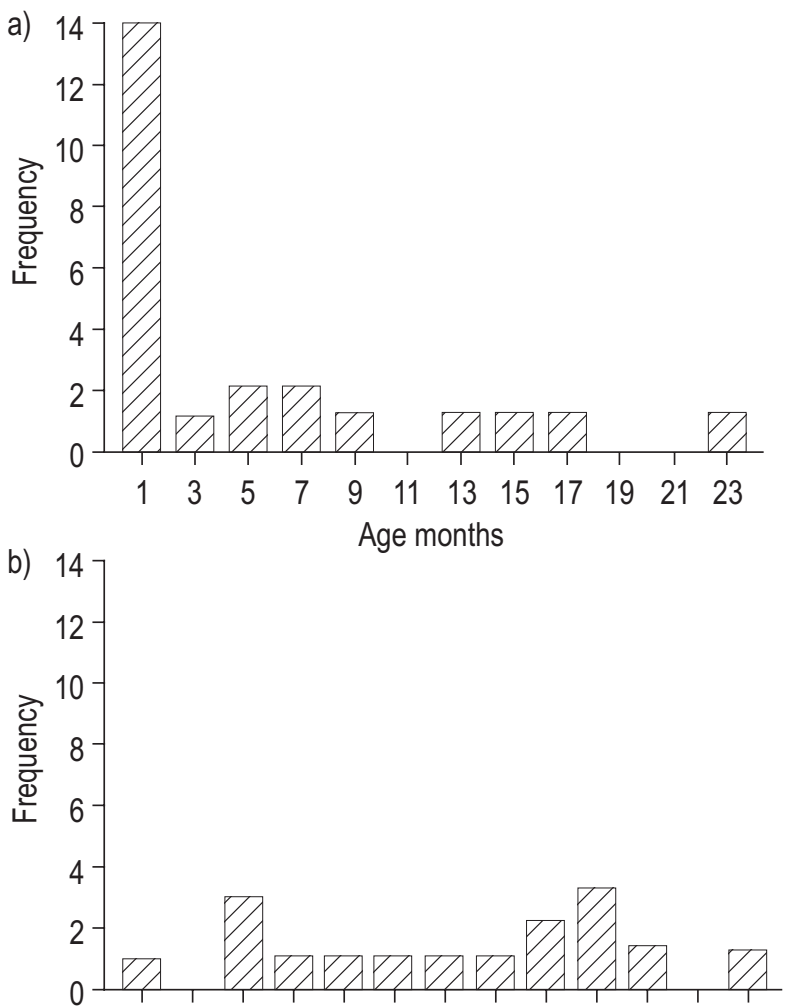

$\begin{array}{lllllllllllll}25 & 29 & 33 & 37 & 41 & 45 & 49 & 53 & 57 & 61 & 65 & 69 & 73\end{array}$

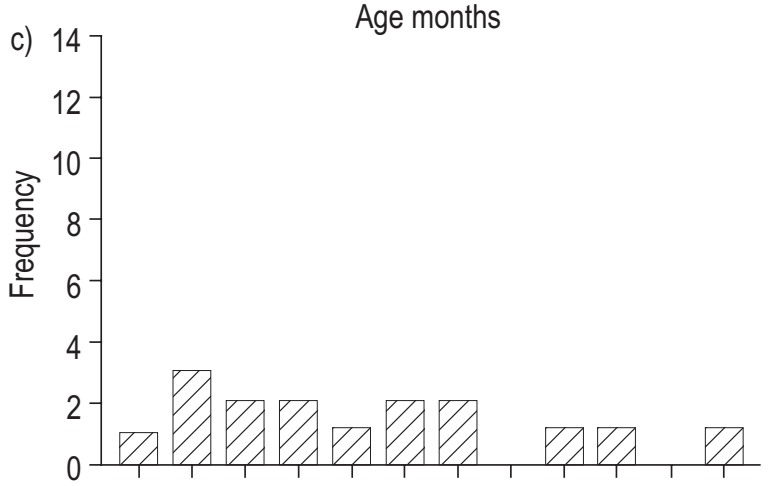

758799111123135147159171183195207 Age months

Fig. 1. - Histogram showing the age distribution of healthy children undergoing bronchoalveolar lavage in a) age group 1 ( $<2$ yrs old $), b)$ age group 2 ( $>2-\leq 5$ yrs old), c) age group $3(\geq 6-\leq 17$ yrs old).

centration $(\mathrm{p}<0.05$ versus group $2, \mathrm{p}<0.01$ versus group 3) (fig. 2d). The neutrophil percentage, albumin-adjusted neutrophil concentration, and eosinophil percentage did not differ between the three groups.

\section{Alveolar macrophage receptor expression}

In the subgroup where AM were used for functional and immunocytochemical studies, there was a trend for a smaller cell area in AM from children $<2$ yrs $(\mathrm{p}=0.06$ versus $\geq 2$ yrs; table 1 ). All paediatric AM stained strongly for CD68, the pan-macrophage marker. The CD68 antigen was distributed in a granular pattern, consistent with its previously reported presence in acid lysosomes [17]. In contrast, CD14 expression, whilst positive on the control monocyte samples, was undetectable on any cells 

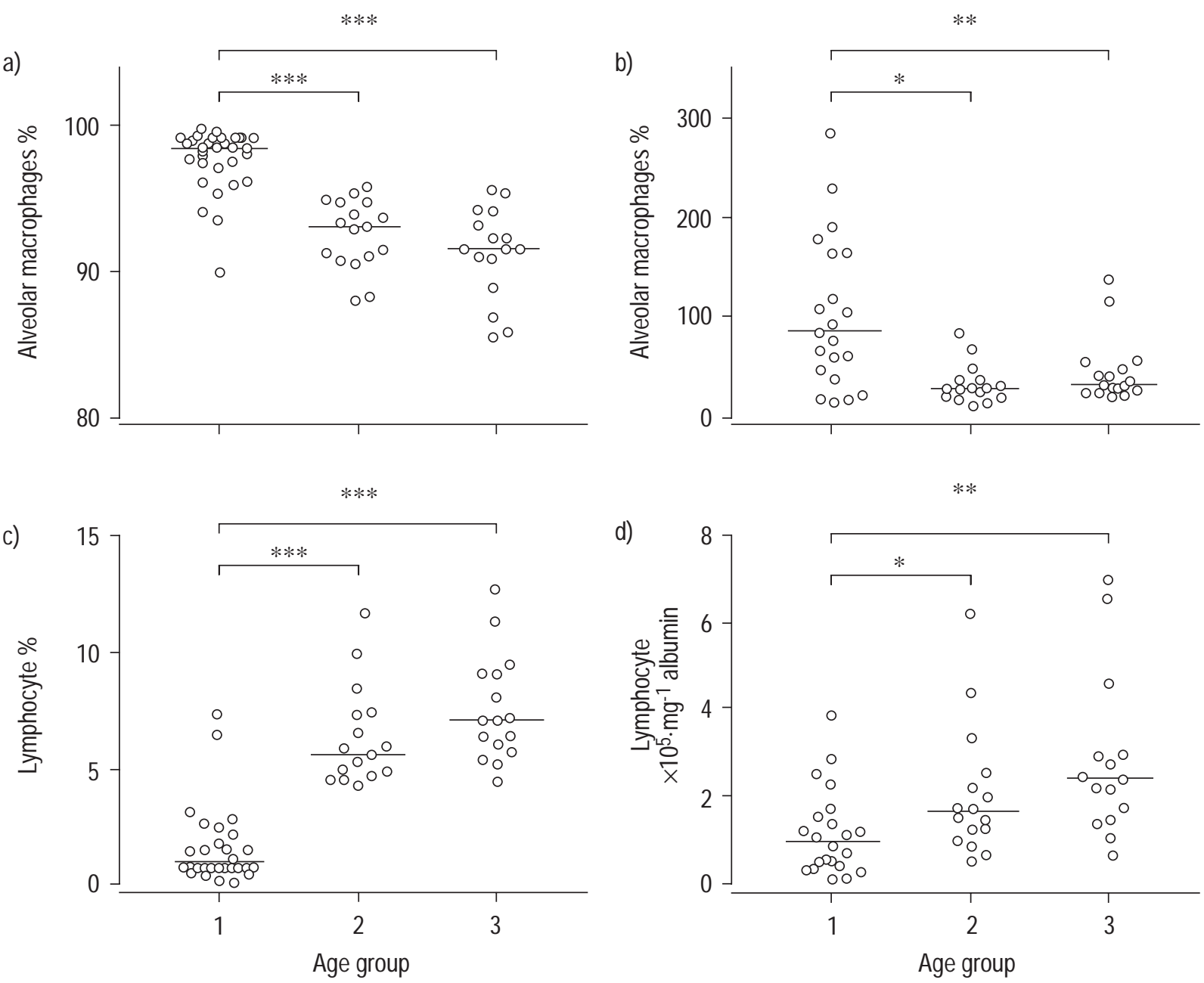

Fig. 2. - Comparison of bronchoalveolar lavage fluid leukocyte parameters between group 1 ( $<2$ yrs), group 2 ( $\geq 2-\leq 5$ yrs) and group 3 ( $\geq 6-\leq 17$ yrs) for: a) alveolar macrophage differential count; b) albumin-adjusted alveolar macrophage concentration; c) lymphocyte differential count; d) albuminadjusted lymphocyte concentration. Horizontal bars represent the median. *: p $<0.05$; **: $\mathrm{p}<0.01$; ***: p<0.0001, versus group 1 (Mann-Whitney U-test).

resembling AM for all children tested. AM from children $<2$ yrs expressed less HLA-DR ( $\mathrm{p}<0.01$ versus $\geq 2$ years) (fig. 3a, table 1). CD54 (intercellular adhesion molecule1) expression did not alter with age (table 1).

\section{Nitro blue tetrazolium reduction}

There was attenuated reduction of NBT by AM from children $<2$ yrs $(\mathrm{p}<0.01$ versus $\geq 2$ yrs, table 1 , fig. $3 \mathrm{~b}$ ). This difference could not be accounted for by the trend for smaller cell size of AM from young children, since the density of NBT reduction per unit area by these AM was also significantly lower than that for AM from older children.

\section{Interleukin-1, -6 and tumour necrosis factor release}

There was no age difference in constitutive release of IL-1, IL-6 and TNF by AM, which was consistently low or undetectable (table 1). On LPS stimulation, AM from children $<2$ yrs released less IL- 1 ( $\mathrm{p}<0.05$ versus $\geq 2$ yrs, fig. $3 \mathrm{c}$, table 1$)$ and less TNF $(\mathrm{p}<0.05$; fig. $3 \mathrm{~d}$, table 1$)$. There was no difference in LPS-stimulated release of IL-6 (table 1).

\section{Alveolar macrophage-stimulation of T-cell proliferation}

Paediatric AM were found to be less effective stimulators of allogeneic T-cells at all doses tested compared to the reference level for adult monocytes. In general, maximal stimulation was achieved at $2.5 \times 10^{3}$ cells $\cdot$ well, with doses of AM above that showing no further increase, and often a decrease in stimulatory capacity. Allogeneic stimulation by AM did not change with age (table 1). AM did support proliferation of anti-CD3 activated T-cells at levels comparable to adult monocytes. Maximal proliferation was achieved using $2.5-5 \times 10^{3}$ AM cells.well, and no developmental change was detected (table 1).

\section{Discussion}

In this study, several deficiencies in AM function during early childhood have been identified, that might alter the response of the immune system to inhaled microbes and antigens. In brief, AM from young children express less HLA-DR release less IL-1 and TNF on stimulation by LPS, and are impaired in their ability to reduce NBT. These differences were associated with increased numbers and percentages of AM in young children. In contrast, there was no developmental change in the capacity of AM to stimulate allogeneic T-cells. 
Table 1. - Comparison of alveolar macrophage (AM) parameters between children $<2$ yrs and $\geq 2$ yrs of age

\begin{tabular}{|c|c|c|c|c|}
\hline AM parameter & $<2$ yrs & $\mathrm{n}$ & $\geq 2 \mathrm{yrs}$ & $\mathrm{n}$ \\
\hline Area AU & $2855(2552,3083)$ & 6 & $3722(2844,3877)$ & 5 \\
\hline CD14 IOD & $\mathrm{ND}$ & 7 & $\mathrm{ND}$ & 5 \\
\hline HLA-DR IOD & $1218(1002,1312)^{*}$ & 7 & $1664(1508,1810)$ & 5 \\
\hline CD54 IOD & $301(236,414)$ & 7 & $340(263,422)$ & 5 \\
\hline NBT reduction IOD & $558(449,653)^{*}$ & 6 & $967(941,1097)$ & 8 \\
\hline \multicolumn{5}{|l|}{ Constitutive cytokine release } \\
\hline IL-1 pg $\cdot 10^{5} \mathrm{AM} \cdot \mathrm{mL}^{-1}$ & $19(10,23)$ & 6 & $25(11,43)$ & 8 \\
\hline $\mathrm{TNF} \mathrm{pg} \cdot 10^{5} \mathrm{AM} \cdot \mathrm{mL}^{-1}$ & $150(47,313)$ & 6 & $62(50,83)$ & 7 \\
\hline IL-6 pg. $10^{5} \mathrm{AM} \cdot \mathrm{mL}^{-1}$ & $39(17,196)$ & 6 & $75(33,346)$ & 8 \\
\hline \multicolumn{5}{|c|}{ LPS-stimulated cytokine release } \\
\hline IL-1 pg $10^{5} \mathrm{AM} \cdot \mathrm{mL}^{-1}$ & $268(172,339)^{\dagger}$ & 6 & $618(412,800)$ & 8 \\
\hline TNF pg $\cdot 10^{5} \mathrm{AM} \cdot \mathrm{mL}^{-1}$ & $125(45,276)^{\dagger}$ & 6 & $500(312,2675)$ & 7 \\
\hline IL-6 pg $10^{5} \mathrm{AM} \cdot \mathrm{mL}^{-1}$ & $1190(585,1515)$ & 6 & $575(462,1405)$ & 8 \\
\hline \multicolumn{5}{|c|}{ Allogeneic T-cell stimulation by AM } \\
\hline nonligated T-cells cpm & $90(72,1475)$ & 7 & $560(158,1516)$ & 11 \\
\hline CD3-ligated T-cells cpm & $951(577,2467)$ & 5 & $1271(604,1818)$ & 9 \\
\hline
\end{tabular}

Data are presented as median (25th, 75 th percentiles); ${ }^{\dagger}: \mathrm{p}<0.05 ;{ }^{\star}: \mathrm{p}<0.01$, (both) versus $\geq 2$ yrs by Mann-Whitney U-test. AU: arbitrary units; IOD: integrated optic density; HLA: human leukocyte antigen; NBT: nitro blue tetrazolium; IL: interleukin; TNF: tumour necrosis factor; LPS: lipopolysaccharide; cpm: counts per minute; ND: not detected.

A marked impairment of the respiratory burst was found, as measured by NBT reduction, in AM from children $<2$ yrs of age. Impaired AM phagocytosis and killing of Candida albicans have been reported in the neonatal monkey [4], and human cord blood monocytes are defective in interferon (IFN)- $\gamma$ induced upregulation of phagocytosis and superoxide release [18]. Deficiencies in NBT reduction by leukocytes have previously been reported, particularly in chronic granulomatous disease. Thus, the impaired production of oxidants by AM in early childhood may contribute to an increased susceptibility to bacterial pulmonary infections. AM from young children were also deficient in their ability to produce IL-1 and TNF, but not IL-6, on LPS stimulation. These cytokines have a critical role in immunomodulation which includes dendritic cell mobilization and maturation [19]. The developmental pattern of IL- 1 and TNF- $\alpha$ release in children is compatible to some, but not all, animal models. For example, neonatal rabbit AM spontaneously release less IL-1- $\alpha$ than adult T-cells, but exhibit increased IL-1 $\alpha$ release with LPS [20]. On the other hand, LPS-stimulated IL- $1 \beta$ release by AM is impaired in the immature rat [21], and in the human several studies have reported the reduced ability of neonatal monocytes to release TNF- $\alpha$ in response to inflammatory stimuli, and their differential ability to release IL-1 and IL-6 [22, 23].

Since no immaturity of LPS-stimulated IL-6 release was observed in group 1, the attenuated LPS-stimulated release of IL-1 and TNF in this group was not due to alterations in cell viability. However, the mechanisms underlying the reduced responsiveness to LPS in AM from young children remain unclear. Whereas blockade of CD14 inhibits LPSstimulated cytokine release from adult macrophages [24], CD14 was not detected on paediatric AM. The possibility that expression was below the sensitivity for the assay cannot be excluded. However, the data suggest that LPS-stimulated cytokine release from AM is not mediated predominantly via surface membrane CD14. Indeed, LPS stimulation of IL-1 and TNF- $\alpha$ release from mononuclear phagocytes does not require CD14 expression [25].

The basis of the decreased expression of HLA-DR on $\mathrm{AM}$ in young children was not determined in the present study, but may be associated with the reported deficiency in IFN- $\gamma$ and IL-4 in early childhood [26]. It has been shown that IL-4 and IFN- $\gamma$ upregulate major histocompatibility complex (MHC) Class II expression on human monocytes [27]; thus, the reduced expression of HLA-DR on cord blood monocytes in comparison to adult monocytes [28], and the immature AM described in the present study, is consistent with the reduced production of IL-4 or IFN- $\gamma$ in early childhood. Despite the increased expression with age of HLA-DR on AM, paediatric AM were found to be uniformly poor stimulators of allogeneic T-cells when compared to the reference adult "large" monocyte population. These results reflect those reported for adult AM [6], and suggest that the ability of AM to present antigen to T-cells does not change during normal development. AM from all children were efficient inducers of CD3-activated T-lymphocyte proliferation under conditions where they were the only source of costimulatory signals. In this circumstance, paediatric AM were as effective as adult monocytes in stimulating T-cells, suggesting that paediatric AM must provide at least those accessory signals sufficient for this form of stimulation. All of the above assays of AM function were conducted on cells which had been frozen under conditions which made no significant change to the assay results when both frozen and fresh peripheral blood monocytes had been compared. Such comparative studies on freshly isolated $\mathrm{AM}$ and frozen replicates were not possible in the present study due to the low number of cells recovered and subject availability.

Immaturity in AM function and immune receptor expression in early childhood was accompanied by a qualitative and quantitative difference in the AM population. The increase in the AM percentage of BALF was confined to group 1 (children $<2$ yrs of age). Thus the AM percentage in BALF in groups 2 and 3 were comparable to that reported for healthy adults [3]. This finding is in agreement with a previous study in older children (4-17 yrs of age) who underwent fibreoptic bronchoscopic BAL prior to elective surgery [29]. The increased AM percentage in the first $2 \mathrm{yrs}$ of life either reflects an increased number of AM per unit lung volume, or a quantitative decrease in 

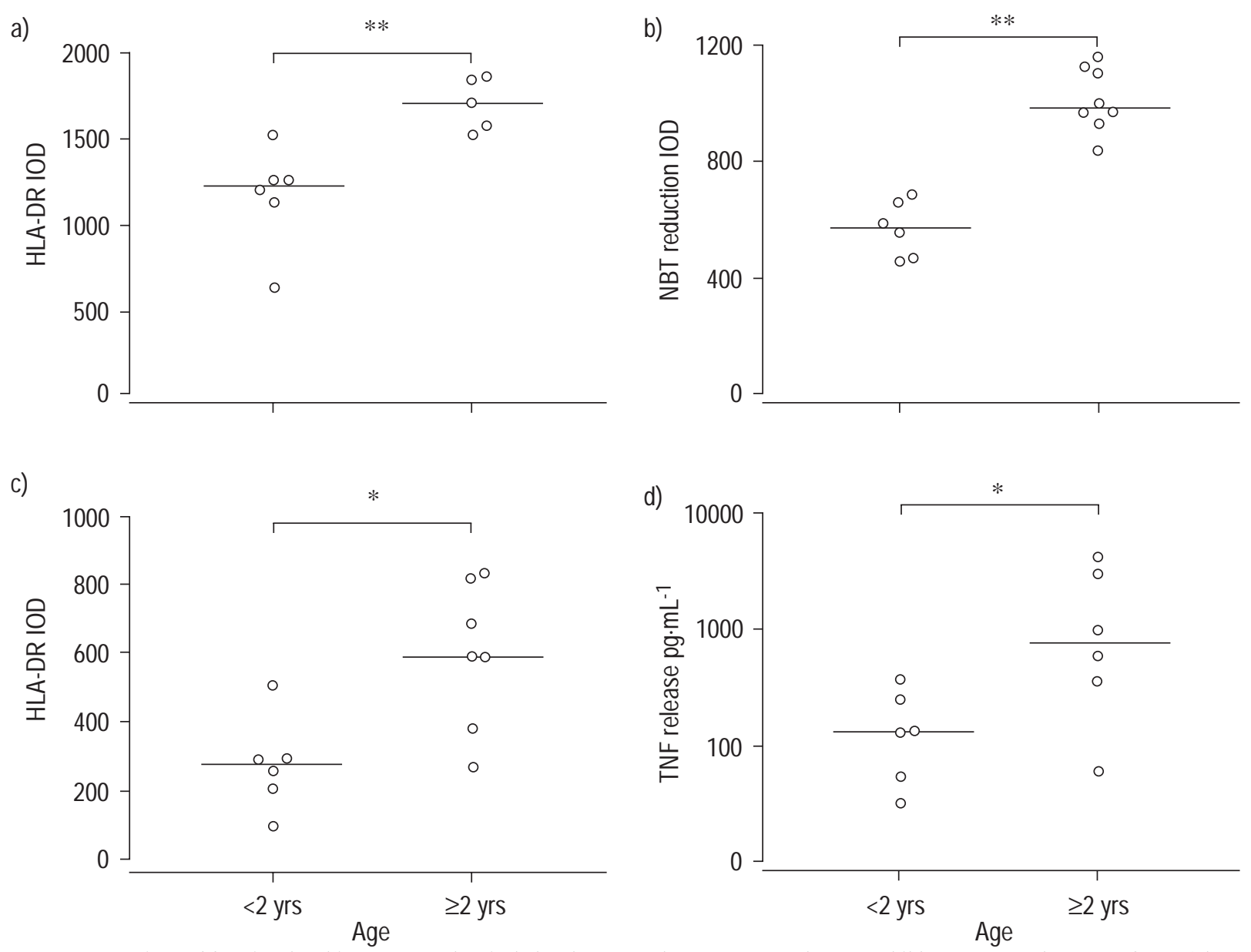

Fig. 3. - Comparison of functional and immunocytochemical alveolar macrophage parameters between children $<2$ yrs and $\geq 2$ yrs of age: a) human leukocyte antigen (HLA)-DR expression expressed as the integrated optic density (IOD); b) nitro blue tetrazolium (NBT) reduction; c) lipopolysaccharide (LPS) stimulated release of interleukin (IL)-1; d) LPS-stimulated tumour necrosis factor (TNF) release. Horizontal bars represent the median. *: $\mathrm{p}<0.05 ; * *: \mathrm{p}<0.01 ; * * *: \mathrm{p}<0.0001$, versus $<2$ yrs (Mann-Whitney U-test).

another leukocyte subpopulation, or a combination of both. Distinguishing between these possibilities is difficult, since the quantitation of BALF leukocytes is particularly susceptible to technical artefacts, and there is no uniformly acceptable method for correcting for dilution. To minimize quantitation errors caused by differences in lavage dynamics across the series, BALF leukocyte concentrations were expressed in two ways: a) indexed to albumin; and b) indexed to the urea-estimated ELF volume (data not shown). Both correction methods, albeit imperfect, indicate that a major determinant of the increased AM percentage in young children is an absolute increase in the number of AM per unit lung volume. The lower albumin-adjusted concentration of lymphocytes in group 1 would also tend to increase the AM proportion and is compatible with a previous observation in a small group of children with stridor, that young children have a lower BALF lymphocyte differential count [30].

To date, there is no histological data to confirm these observations. However, the developmental changes in the human mirror those reported in some animal models. In the sheep, the number of AM falls progressively from a peak during the first post-natal week to a nadir at full maturity, and the number of lymphocytes increases slowly over the same period [31]. Increased recruitment of a "monocytic" population from the systemic circulation in early life could account for both the increase in AM number and the functional and phenotypic differences during this period. Indeed, the prenatal period is associated with a massive expansion of the rabbit AM population, but it is unclear whether this reflects an influx of monocytes [32]. The smaller AM seen in young children may imply increased levels of monocyte recruitment from the systemic circulation. In this case, AM would express a phenotype similar to cord monocytes. There are functional differences between cord and adult monocytes [22, 28], and some of the phenotypes reported for neonatal monocytes are reminiscent of those that have been described for AM in children $<2$ yrs of age. Since we found no evidence of surface staining of the monocyte marker CD14 on AM, a candidate precursor may be the blood monocyte population which is low in CD14 expression and positive for CD16 [33]. Like AM from children less than 2 years of age, this subpopulation of adult monocytes is also deficient in its ability to reduce NBT, but strongly expresses HLA-DR and several adhesion molecules [33].

In conclusion, this study has highlighted quantitative and functional differences in the alveolar macrophage population in early childhood. In particular, the altered alveolar leukocyte differential, deficient release of tumour necrosis factor and interleukin-1, and attenuated nitro blue 
tetrazolium reduction by alveolar macrophage from young children, may contribute to an altered alveolar milieu in the first years of life. In contrast, no evidence of enhanced Tcell stimulation by alveolar macrophage in early childhood was found that would contribute to an increased vulnerability to antigen sensitization was found.

Acknowledgements. The authors thank E. Uren for performing the viral cultures.

\section{References}

1. Holt PG. Postnatal maturation of immune competence during infancy and childhood. Pediatr Allergy Immunol 1995; 6: 59-70.

2. Holt PG. Immune tolerance and protection against allergic sensitization. Allergy 1995; 50: 34-36.

3. Anonymous. Bronchoalveolar lavage constituents in healthy individuals, idiopathic pulmonary fibrosis, and selected comparison groups. The BAL Cooperative Group Steering Committee. Am Rev Respir Dis 1990; 141: S169-S202.

4. Kurland G, Cheung AT, Miller ME, Ayin SA, Cho MM, Ford EW. The ontogeny of pulmonary defences: alveolar macrophage function in neonatal and juvenile rhesus monkeys. Pediatr Res 1988; 23: 293-297.

5. Spiteri MA, Poulter LW. Characterization of immune inducer and suppressor macrophages from the normal human lung. Clin Exp Immunol 1991; 83: 157-162.

6. Toews GB, Vial WC, Dunn MM, et al. The accessory cell function of human alveolar macrophages in specific T-cell proliferation. J Immunol 1984; 132: 181-186.

7. Grigg J, Kleinert S, Woods RL, et al. Alveolar epithelial lining fluid cellularity, protein and endothelin-1 in children with congenital heart disease. Eur Respir J 1996; 9: 1381-1388.

8. Rennard SI, Basset G, Lecossier D, et al. Estimation of volume of epithelial lining fluid recovered by lavage using urea as marker of dilution. J Appl Physiol 1986; 60: 532-538.

9. Uren S, Boyle W. Stimulation of allogeneic and autologous MLR by subpopulations of human monocytes. Transplant Proc 1989; 21: 208-210.

10. Hancock WW, Sayegh MH, Sablinski T, Kut JP, KupiecWeglinski JW, Milford EL. Blocking of mononuclear cell accumulation, cytokine production, and endothelial activation within rat cardiac allografts by CD4 monoclonal antibody therapy. Transplantation 1992; 53: 1276-1280.

11. Rook GA, Steele J, Umar S, Dockrell HM. A simple method for the solubilisation of reduced NBT, and its use as a colorimetric assay for activation of human macrophages by gamma-interferon. J Immunol Methods 1985; 82: 161-167.

12. Gearing AJ, Bird CR, Bristow A, Poole S, Thorpe R. A simple sensitive bioassay for interleukin-1 which is unresponsive to 10(3) U/ml of interleukin-2. J Immunol Methods 1987; 99: 7-11.

13. Van Snick J, Cayphas S, Vink A, et al. Purification and NH2-terminal aTnino acid sequence of a T-cell-derived lymphokine with growth factor activity for B-cell hybridomas. Proc Natl Acad Sci USA 1986; 83: 9679-9683.

14. Espevik T, Nissen-Meyer J. A highly sensitive cell line, WEHI 164 clone 13, for measuring cytotoxic factor/tumor necrosis factor from human monocytes. $J$ Immunol Methods 1986; 95: 99-105.

15. Hansen MB, Nielsen SE, Berg K. Re-examination and further development of a precise and rapid dye method for measuring cell growth/cell kill. J Immunol Methods 1989; 119: 203-210.

16. O'Brien J, Knight S, Quick NA, Moore EH, Platt AS. A simple technique for harvesting lymphocytes cultured in Terasaki plates. J Immunol Methods 1979; 27: 219-223.

17. Betjes MG, Haks MC, Tuk CW, Beelen RH. Monoclonal antibody EBM1 1 (antiCD68) discriminates between dendritic cells and macrophages after short-term culture. Immunobiology 1991; 183: 79-87.

18. Marodi L, Kaposzta R, Campbell DE, Polin RA, Csongor J, Johnston RB Jr. Candidacidal mechanisms in the human neonate. Impaired IFN-gamma activation of macrophages in newborn infants. J Immunol 1994; 153: 5643-5649.

19. Lanzavecchia A. Mechanisms of antigen uptake for presentation. Curr Opin Immunol 1996; 8: 348-354.

20. Rozycki HJ. Elevated production of interleukin-1-beta from alveolar rnacrophages isolated from newborn rabbits. Biol Neonate 1994; 66: 93-99.

21. Bakker JM, Broug-Holub E, Kroes H, Rees EP, Kraal G, Iwaarden JF. Functional immaturity of rat alveolar macrophages during postnatal development. Immunology 1998; 94: 304-309.

22. Peters AM, Bertram P, Gahr M, Speer CP. Reduced secretion of interleukin-1 and tumor necrosis factor-alpha by neonatal monocytes. Biol Neonate 1993; 63: 157-162.

23. Pillay V, Savage N, Laburn H. Circulating cytokine concentrations and cytokine production by monocytes from newborn babies and adults. Pflugers Arch 1994; 428: 197-201.

24. Dentener MA, Bazil V, von Asmuth EJ, Ceska M, Buurman WA. Involvement of CD14 in lipopolysaccharide-induced tumor necrosis factor-alpha, IL-6 and IL-8 release by human monocytes and alveolar macrophages. $J$ Immunol 1993; 150: 2885-2891.

25. Lynn WA, Liu Y, Golenbock DT. Neither CD14 nor serum is absolutely necessary for activation of mononuclear phagocytes by bacterial lipopolysaccharide. Infect Immun 1993; 61: 4452-4461.

26. Tang ML, Kemp AS, Thorburn J, Hill DJ. Reduced interferon-gamma secretion in neonates and subsequent atopy. Lancet 1994; 344: 983-985.

27. Hart PH, Bonder CS, Jones CA, Finlay-Jones JJ. Control of major histocompatibility complex class II expression on human monocytes by interleukin-4 regulatory effect of lipopolysaccharide. Immunology 1996; 89: 599-605.

28. Stiehm ER, Sztein MB, Steeg PS, et al. Deficient DR antigen expression on human cord blood monocytes: reversal with lymphokines. Clin Immunol Immunopathol 1984; 30: 430-436.

29. Ratjen F, Bredendiek M, Brendel M, Meltzer J, Costabel U. Differential cytology of bronchoalveolar lavage fluid in normal children. Eur Respir J 1994; 7: 1865-1870.

30. Riedler J, Grigg J, Stone C, Tauro G, Robertson CF. Bronchoalveolar lavage cellularity in healthy children. Am J Respir Crit Care Med 1995; 152: 163-168.

31. Weiss RA, Chanana AD, Joel DD. Postnatal maturation of pulmonary antimicrobial defense mechanisms in conventional and germ-free lambs. Pediatr Res 1986; 20: 496504.

32. Zeligs BJ, Nerurkar LS, Bellanti JA, Zeligs JD. Maturation of the rabbit alveolar macrophage during animal development. I. Pennatal influx into alveoli and ultrastructural differentiation. Pediatr Res 1977; 11: 197-208.

33. Uren S, Boyle W. Isolation of a small monocyte subset with high accessory function: a dendritic cell precursor? Transplant Proc 1995; 27: 2172-2173. 